\title{
The role of mass media in disease outbreak reporting in the United Republic of Tanzania
}

\author{
L.E.G. MBOERA \& S.F. RUMISHA \\ National Institute for Medical Research, Dar es Salaam, Tanzania
}

\begin{abstract}
Emerging infectious diseases and the growth of information communication technology have produced new demands and possibilities for disease outbreak information and reporting. An analysis of disease outbreak information and reporting by the Tanzanian mass media was carried out to cover a 12-month period between July 2002 and June 2003. Outbreak episodes reported in four local dailies were collected, compiled and analysed. The disease that received the highest coverage was cholera. Cholera outbreaks were frequently reported from the 3 districts in Dar es Salaam Region. Other epidemic-prone diseases received very little coverage. These included meningitis and rabies. With globalisation and high technological information systems, reports of disease outbreaks are more widely disseminated and more easily accessible than ever before. However, the quality of information is no longer controlled and may be provided out of context, often causing unnecessary public anxiety and confusion. It is important that mass media is provided with accurate epidemiological reports if correct information is to reach the public. The role of media in outbreak reporting is herein discussed in relation to the importance of providing timely and accurate epidemiological information.
\end{abstract}

\section{Introduction}

An epidemic may be defined as an occurrence in a community or region of cases of an illness or healthrelated events clearly in excess of normal expectancy. In Tanzania, epidemics are usually reported upon confirmation of the disease and when the disease surpasses an epidemic threshold. Under the current integrated disease surveillance and response strategy epidemic-prone diseases in Tanzania include bacillary dysentery, cholera, meningitis, measles, plague, rabies and yellow-fever (MoH, 2001). However, malaria epidemics are not uncommon and have been reported in a number of districts (Mboera and Kitua, 2001; Mboera, 2004).

The rapid global expansion of telecommunications and broadened access to news media and the Internet have changed the way society treats information, especially as regards to disasters. Globalisation presents new challenges and opportunities in combating diseases likely to cause epidemics (Grein et al., 2000). As a result of increased international travel and trade, local events acquire international importance. In many cases, either local leaders or the mass media report disease outbreaks as rumours. Outbreak rumours that later prove to be unsubstantiated may lead to inappropriate response, causing disruption in travel and trade and economic loss of affected countries. Suspected reported outbreaks or rumours need to be verified as quickly as possible so that expensive resource is not utilised for investigation and response. To avoid these unnecessary expenses and travel disturbance, all outbreaks should be assessed in terms of their potential to spread and their national and international importance.

In Tanzania, it is the responsibility of the Ministry of Health to systematically gather and assess information on suspected outbreaks in the form of epidemic intelligence from a variety of sources, most frequently, District Medical Officers and Regional Medical Officers. However, outbreak rumours reported by others need to be investigated and verified. Verification of outbreaks will usually be sought through the District Medical Officers. The Ministry of Health usually, verifies and confirms the existence of an outbreak in a timely manner. In principle, after timely detection of a suspected outbreak, an outbreak investigation is done to confirm the presence of an outbreak and to characterize three elements essential to the development of control strategies: cause, source and progression. 
All reports of suspected outbreaks in Tanzania must be reported within 24 hours and investigated within 48 hours $(\mathrm{MoH}, 2001)$. When the district outbreak investigation (rapid response) team receives an unconfirmed outbreak report, the relevance to national or international public health is assessed, and, if appropriate, further information is sought. Outbreak verification is based on a broad range of information sources, including newspapers, television, radio, and verbal reports by community or local leaders. Usually, the district outbreak investigation team will establish the potential importance of the event, on the basis of available background information, endemicity levels, and details of previous outbreaks and will report to the National level through the Regional authorities.

Timely information of outbreak to those who need to know is a key aspect of the outbreak verification process, and details of outbreaks with potential for international public health importance are disseminated through various means. Information is shared directly with all stakeholders for immediate action.

In this paper, an analysis of disease outbreak information and reporting by the Tanzanian mass media was carried out to cover a 12-month period between July 2002 and June 2003. The objective was to assess the role of mass media in outbreak reporting as a means of informing the public on epidemic-prone disease situation in the country.

\section{Materials and methods}

This study was conducted as a desk review of disease outbreak information that was reported by the
Tanzanian local dailies during a 12-month period between July 2002 and June 2003. Information on outbreak that was covered in four local dailies was collected, compiled and analysed. The dailies from which information was collected included:

Nipashe, Mwananchi, Majira and Mtanzania. These newspapers are published daily in Kiswahili, a national language in Tanzania. Each day, the newspapers were searched for any news on disease outbreak. Outbreak events were then recorded in pre-designed matrix to capture the following information: type of disease, place of occurrence (district), date of occurrence, date of reporting, number of people affected, number of deaths and any measure taken to contain the outbreak.

\section{Results}

Ninety-nine reports on outbreaks were covered by the 4 dailies during the 12-month period. Most (53.5\%) of the outbreaks were reported by the Mwananchi, followed by Nipashe and Majira, which accounted for $19.2 \%$ and $18.2 \%$ of the reports, respectively. Reports in the Mtanzania accounted for only $8.1 \%$.

Disease outbreaks reported by the mass media included cholera, meningitis and rabies. Cholera was the most frequently reported outbreak, accounting for $85.2 \%$ of all of the reports covered in the newspapers. Cholera outbreaks were reported in $18.2 \%(22 / 121)$ of the districts of Tanzania mainland. Two reports of cholera outbreak were from Zanzibar. The districts affected by cholera during the period of study are shown in Table 1. 
Table 1: Cholera outbreaks as reported by Media in Districts of Tanzania

\begin{tabular}{llll}
\hline District & No. of reports & No. of months reported & Source \\
\hline Ilala & 37 & 10 & Nipashe, Mwananchi, Mtanzania, Majira \\
Kinondoni & 28 & 10 & Nipashe, Mwananchi, Mtanzania, Majira \\
Temeke & 18 & 10 & Nipashe, Mwananchi, Majira \\
Rungwe & 1 & 1 & Nipashe \\
Kyela & 1 & 1 & Nipashe, Mwananchi \\
lleje & 1 & 1 & Nipashe \\
Bagamoyo & 3 & 2 & Mwananchi, Majira \\
Morogoro & 5 & 4 & Nipashe, Mwananchi, Majira \\
Kilombero & 3 & 2 & Nipashe, Mtanzania, Mwananchi \\
Kilindi & 1 & 1 & Mwananchi \\
Kibaha & 2 & 2 & Mtanzania, Majira \\
Dodoma Rural & 1 & 1 & Nipashe \\
Dodoma Urban & 4 & 3 & Mtanzania, Majira \\
Ujiji & 1 & 1 & Radio One \\
Korogwe & 3 & 3 & Nipashe, Majira \\
Chunya & 1 & 1 & Mtanzania \\
Mvomero & 1 & 1 & Mwananchi \\
Kilosa & 2 & 2 & Nipashe, Majira \\
Tanga Urban & 1 & 1 & Nipashe \\
Kiteto & 1 & 1 & Majira \\
Manyoni & 1 & 1 & Mwananchi \\
Tunduru & 2 & 1 & Nipashe, Majira \\
Zanzibar & 2 & 1 & Mtanzania, Majira \\
\hline & & & \\
\hline
\end{tabular}

Interestingly, most of the reports were from Ilala, Temeke and Kinondoni districts in Dar es Salaam. Ilala was the most affected district. Nine of the reports from Dar es Salaam Region did not indicate the affected specific district. Of these unspecified outbreaks, Mwananchi reported 6 outbreaks, Majira 2 Nipashe and Mtanzania 1 outbreak each. Reports of outbreaks were covered throughout the 12-month period (except September 2002 and February 2003) in Ilala, Kinondoni and Temeke districts. For the other 20 districts affected by cholera, outbreaks were reported mainly in October 2002 (7) and December 2002 (5). Of the outbreaks reported from Dar es Salaam, Nipashe, Mwananchi, Mtanzania and Majira reported $42.1 \%, 86.8 \%, 25 \%$ and $33.3 \%$, respectively. Majira and Mtanzania covered most of the reports from the areas outside Dar es Salaam.

Other diseases or conditions reported by the mass media included rabies, meningitis and dog bites. During the year under study, Nipashe reported rabies from Mvomero district and Morogoro Municipality. Dog bites were reported in Morogoro (April 2003), Dodoma rural (October 2002) and Kilombero districts (May 2003). Meningitis was reported to occur in Mbozi (October 2002) and Kigoma (July 2002).

\section{Discussion}

Outbreaks of communicable diseases are commonly experienced in most districts of Tanzania (Rumisha et al., 2003). In 2002 and 2003 communicable diseases such as cholera, meningitis, bacillary dysentery, measles, acute flaccid paralysis and rabies continued to be the major public health problems in the country (MoH, unpublished). Of all the epidemic-prone diseases, cholera accounts for the highest proportion of the number of outbreaks in Tanzania causing considerable socio-economic cost to affected populations. Fifteen regions $(75 \%)$ reported cases of cholera at different times of 2002 and 2003. A total of 11,625 cases of cholera and 298 deaths due to were 
reported in 2002 alone. It is not surprising therefore that the local dailies covered more reports of cholera than any of the other outbreaks.

The frequency of occurrence of cholera in the districts of Dar es Salaam reported by the mass media is substantiated by data from Ministry of Health and our previous investigations. During recent years, cholera has been a common health problem in Ilala District (Mayala et al., 2003). For instance, in 1997, a total number of 3,220 cases and 128 deaths due to cholera were reported in Ilala district alone (DEHP, 1998). Findings of a recent study in Ilala indicate that cholera cases have been consistently reported in the district since 1997 (Mayala et al., 2003). Cholera has now become endemic to Tanzania with small outbreaks being reported every year and a much larger outbreak occurring every 4-5 years (WHO, 2002).

Although the mass media reported the occurrence of meningitis in Mbozi and Kigoma, data from the Ministry of Health, indicates that all regions except Mtwara and Iringa experienced outbreaks of the disease during 2002. Serious meningitis outbreaks occurred in Kigoma, Kagera, Shinyanga and Tabora regions. The Ministry of Health data for the year 2002 shows a total of 1,598 cases and 290 deaths due to meningitis were reported to have occurred in Tanzania.

Although rabies is among the diseases under the current integrated disease surveillance and response strategy in Tanzania, it is still considered to be a relatively insignificant human disease, accounting for only $1 \%$ of deaths attributable to infectious diseases in the world. However, the number of animal bites has been increasing year after year. Its low reporting coverage is probably because of the fact that it is usually neglected and given little attention. Nonetheless, the Ministry of Health data available for the year 2002 indicate that 9,606 cases of dog bites and 10 deaths were reported from the Regions $(\mathrm{MoH}$, 2002).

Despite the fact that a low proportion of disease outbreak is reported by the mass media, particularly, newspapers, these media play a great role in informing the public of the occurrence of disease outbreaks in the country. It has happened that sometimes it is the media, which provides the first information, though as rumours, about an outbreak occurrence. It is therefore the responsibility of the authorities concerned to act immediately on rumours or reports of suspected outbreaks that are provided by our local mass media. However, increasing numbers of outbreak reports must be assessed rapidly so that control efforts can be initiated and unsubstantiated reports identified so as to protect the country from unnecessary socioeconomic damage.

Although it is expected that all reported outbreak rumours must be verified, some of these reports are not acted upon by the relevant authority until when the outbreak is already out of control. It has been observed that often there are delays in reporting of cases of epidemic prone diseases when they occur. This has happened during the malaria outbreaks in Lushoto in 1997 and Muleba in 1998 (Mboera, 2004). Untimely response to outbreaks may results in catastrophic situations with high mortalities which otherwise could have been prevented. Not only that response to rumours through the local media or community are not investigated, feedback from the national level to the regions/districts and to the lower levels on reported outbreaks is lacking.

It was interesting to observe that most of the outbreaks were reported from the 3 districts of Dar es Salaam. This was not surprising for the fact that the daily newspapers are based in Dar es Salaam, and collecting and disseminating information is probably easier than from the countryside. In addition, reports of cholera that was frequently experienced in the three districts attracted most of the attention of the media and the community at large.

In conclusion, effective disease control requires prompt and adequate action towards reduction or elimination of existing conditions, and preventing the occurring of new ones. Such actions can only be made if correct information reaches those required to take action timely. Mass media may sometimes be the first to access and disseminate information on disease outbreak. However, the quality of information they collect and disseminate is no longer controlled and may be provided out of context, often causing 
unnecessary public anxiety and confusion. It is important that mass media is provided with accurate epidemiological reports if correct information is to reach the public. It is essential to strengthen capacity of our journalists in health and disease reporting skills to avoid misreporting. In Tanzania, already advocacy through the media has been identified as one of the most effective ways of delivering information to the targeted audience. The media serves as a conduit of information in a two-way flow between the Ministry of Health or Government and the targeted community (Ijumba et al., 2003). The national and district level authorities are therefore urged to provide accurate and timely information on disease outbreaks so that correct epidemiological information is timely provided to the public. Such information is likely to raise awareness of the disease and hence facilitate community mobilisation in outbreak response. Provision of information on occurrence of disease outbreak is also likely to alert the community and prepare them for an impending disaster. Epidemic preparedness includes measures taken to enhance the abilities of individuals, communities, districts and the nation to respond to disease outbreaks.

\section{References}

Grein, T.W., Kamara, K.O., Rodier, G., Plant, A.J., Bovier, P., Ryan, M.J., Ohyama, T. \& Heymann, D.L. (2000) Rumors of disease in the global village: outbreak verification. Emerging Infectious Diseases 6 (2), MarchApril 2000.

DEHP (1998) Dar es Salaam Environmental Health Programme. Dar es Salaam City Council, Tanzania.
Ijumba, J.N., Dawson, A., Lloyde, C., Ross, L., Hommel, M. \& Kitua, A.Y. (2003) Malaria and the media: advocating a healthy policy and practice. Tanzania Health Research Bulletin 5, 30.

Majira (July 2002- June 2003). Business Times Publications.

Mayala, B.K., Mboera, L.E.G. \& Gwacha, F. (2003) Mapping cholera risks using Geographical Information System in Ilala District, Tanzania. Tanzania Health Research Bulletin 5, 8-12.

Mboera, L.E.G. (2004) Environmental and socioeconomic determinants of malaria epidemics in the highlands of Tanzania. Tanzania Health Research Bulletin 6, 11-17.

Mboera, L.E.G. \& Kitua, A.Y. (2001) Malaria epidemics in Tanzania; An overview. African Journal of Health Sciences 8, 14-18.

Mtanzania (July 2002- June 2003). Habari Corporation Limited.

Mwananchi (July 2002- June 2003), Mwananchi Communication Limited.

Nipashe (July 2002- June 2003), The Guardian Limited.

Rumisha, S.F., Mboera, L.E.G. Kamugisha, M.L., Kalumuna, A., Amri, M. \& Kitua, A.Y. (2003) Pattern and distribution of communicable diseases in border districts of Bukoba and Tanga, Tanzania. Tanzania Health Research Bulletin 5, 19-23.

WHO (2002) Annual Report on WHO Country Office Operational Activities in the Health Sector 2001. WHO, Dar es Salaam, Tanzania. 


\section{Notice to contributors}

Manuscripts submitted to Tanzania Health Research Bulletin should be original research papers on health. Short review (by prior arrangement with the editor), opinion papers, short communications and technical reports may also be accepted.

Papers should be in clear concise English, normally not exceeding 6000 words of text (12, A4 printed pages) but longer papers of particular merit may be accepted. Papers submitted must not have been published or be under consideration for publication by any other journal.

The title of the paper should be informative, but preferably not exceeding twenty words. The paper should include a self-contained abstract, in one paragraph, of less than 250 words, presented as a series of factual statements to follow under the title.

The name and address of the author to whom readers should address correspondence should be given on the first page. Manuscripts must be typed, on one side of the paper, double-spaced with wide margins, preferably on A4 or quarto paper. Tables must be simple, on separate sheets, and should be selfexplanatory. Tables should not be used where text or illustrations give the same information. Figure legend should be grouped together on a separate sheet. A hard copy and diskette containing the manuscript are required.
References should conform to the "name-andyear' system, give full journal titles and conform to the following styles:

Powell, W. (1986) Enhancing parasitoid activity in crops. Pp. 319-340 in Waage, J. \& Greathead, D. (eds) Insect Parasitoids. London, Academic Press (Symposium, Royal Entomological Society of London No. 13).

Matola, Y.G. (1985) Prospects of human malaria and bancroftian filariasis infections in the Lower Rufiji Basin, Tanzania I. Malaria. Tropical and Geographical Medicine 37, 102-107.

The general public, policy and decision makers and scientists are particularly welcome to submit short articles and letters for publication in the Bulletin. The letters should not exceed one page, and may comment on any article previously published in the Bulletin, or on perceived problems in the health sector requiring researched solutions.

All correspondences on Tanzania Health Research Bulletin should be addressed to Dr. L.E.G. Mboera, National Institute for Medical Research, Ocean Road, P.O. Box 9653, Dar es Salaam, Tanzania. Email: lmboera@nirm.or.tz, 1mboera@hotmail.com. 